continuity of primary care for many, as well as strengthen and accelerate the re-establishment of healthcare in the postdisaster period.

Prehosp Disaster Med 2017;32(Suppl. 1):s72-s73

doi:10.1017/S1049023X17001935

\section{The Role of Hospital Volunteers in Disaster Planning and Response \\ Alexandra Bernard}

Volunteer Services, McGill University Health Centre, Montreal/Canada

Study/Objective: Discuss hospital volunteers in disaster planning and response in a large, urban tertiary care center.

Background: Volunteer Services at The McGill University Health Centre (MUHC) began participating in disaster response and training following the 1989 École Polytechnique massacre in Montreal, Canada. Over time the program has grown and is now comprised of over 80 volunteers across two hospitals.

Methods: The MUHC volunteers were deployed in 2 official emergency situations (Concordia University massacre in 1992 \& Dawson College shooting in 2006), and provided support during the January 1998 North American ice storm. In addition, they participate in hospital emergency planning meetings, yearly disaster response training including live mass casualty simulations, and fan-out testing drills. The MUHC Volunteer Department has provided 10 to 80 simulated patients for each of the training exercises conducted since 2012. Results: Through participation in disaster planning and response, hospital volunteers were found to be a reliable, well trained, independent, un-costly and flexible source of manpower, familiar with the hospital environment and functioning. Specific roles have been identified and integrated into the external disaster response plan:

- Assist ED Nursing and Security teams, directing patients to designated disaster triage entrance

- Assist ambulatory patients to treatment areas after ED triage

- Perform regular ED Duties with an expected increased workload

- Participate in the Psychosocial Response with other members of the multidisciplinary Psychosocial Response Team

- Perform regular inventory of disaster response materials Frequent turnover and limited funds for training are factors limiting further involvement of hospital volunteers in emergency management.

Conclusion: Hospital volunteers are a valuable asset to the hospital during an external disaster. Their involvement in emergency management activities including regular attendance at planning meetings and participation in simulations are key to successful collaboration when external disasters occur.

Prehosp Disaster Med. 2017;32(Suppl. 1):s73

doi:10.1017/S1049023X17001947

\author{
Catastrophic Data Disruptions: A New Frontier for \\ Disaster Preparedness \\ Puneet Seth \\ Emergency Medicine, Singhealth (Sengkang Hospital), Singapore/ \\ Singapore
}

Study/Objective: To outline the causes, effects, and mitigation strategies of catastrophic health care data disruption.

Background: Recent years have seen a surge in disruption of health care delivery due to various causes. Health care data has become a target for a variety of actors, especially in the field of cyberspace. As we become more urbanized, we have increasingly become dependent on information technology to retain and retrieve long and complicated patient medical records to allow us to practice medicine safely and efficiently. Thus, the cyber-physical system has become a key infrastructure item that needs to be protected against threats.

Methods: A detailed Internet search was conducted to look for possible causes, effects, and mitigation strategies of catastrophic data disruption. The findings were listed in the results.

Results: Risks to Patient Data Systems emanate from: 1. Targeted cyber-attacks:

- Disgruntled employees;

- Cybercriminals- especially for Ransomware;

- Nation states.

2. Business risk to any part of the data chain:

- Attack on database provider's cloud;

- Financial bankruptcy of any part of the chain (vendor/ sub-vendor);

- Physical infrastructural damage to servers/storage systems.

3. Power outage and related IT failure. 4. Solar flares. 5. EMP weapons. Immediate effects on health care delivery are: 1. Cascading failure of health delivery. 2. No medical records can be retrieved, bringing care to a halt. 3. Medications cannot be served safely. 4 . Impaired care of patients on critical care support - ICUs, EDs, hemodialysis, and chemotherapy units. 5. No refilling of prescriptions. Best Practices in Operations Continuity involve: 1 . Crisis Communications protocol for a confirmed outage. 2. Scenario-based preparedness exercises: Downtime Protocol. 3. Easy-to-deploy paper-based recording material: Downtime toolkit. 4. Service recovery with alternative offsite hardware and software providers. 5. Cloud-based backup redundancy arrangements.

Conclusion: Catastrophic Data Disruptions are a new frontier in disaster preparedness. It involves everyone and can be crippling if not prepared for in advance.

Prehosp Disaster Med. 2017;32(Suppl. 1):s73

doi:10.1017/S1049023X17001959

Ensuring Operational Continuity of Community Healthcare Services During Disasters

Gal Horowitz

Medical Quality Control, Emergency And Disaster Management, kupat holim meuhedet, Tel- Aviv Yafo/Israel

Study/Objective: To present contribution of a national guideline on the capacity of community primary care health care systems to establish and sustain operational continuity during disasters.

Background: "Meuhedet" is the $3^{\text {rd }}$ largest Health Maintenance Organization (HMO) in Israel, insuring $15 \%$ of the population ( 1,200,000 members). The HMO is committed to 